\title{
Automation of Optimized Gabor Filter Parameter Selection for Road Cracks Detection
}

\author{
Haris Ahmad Khan \\ Laboratoire Electronique, \\ Informatique et Image (Le2i) \\ Université de Bourgogne \\ Dijon, France
}

\author{
M. Salman, Sajid Hussain \\ Wah Engineering College, \\ University of Wah \\ Wah Cantt, Pakistan
}

\author{
Khurram Khurshid \\ Electrical Engineering Department \\ Institute of Space Technology \\ 1, Islamabad highway, Islamabad, \\ 44000, Pakistan
}

\begin{abstract}
Automated systems for road crack detection are extremely important in road maintenance for vehicle safety and traveler's comfort. Emerging cracks in roads need to be detected and accordingly repaired as early as possible to avoid further damage thus reducing rehabilitation cost. In this paper, a robust method for Gabor filter parameters optimization for automatic road crack detection is discussed. Gabor filter has been used in previous literature for similar applications. However, there is a need for automatic selection of optimized Gabor filter parameters due to variation in texture of roads and cracks. The problem of change of background, which in fact is road texture, is addressed through a learning process by using synthetic road crack generation for Gabor filter parameter tuning. Tuned parameters are then tested on real cracks and a thorough quantitative analysis is performed for performance evaluation.
\end{abstract}

Keywords-Pavement Cracks; Automated detection; Gabor Filters; Genetic Algorithm; Parameter Selection

\section{INTRODUCTION}

Transportation structure is built around pavements and roads. These roads require periodic health inspections so that information concerning their physical condition can be gathered and recommendations can be made for their reconstruction or repair if required. In time detection of cracks, which is pivotal in executing economical pavement maintenance thus avoiding more damage, both physical and financial. The topmost layer of the flexible pavement is known as asphalt course which is constructed by black bitumen and one down size aggregate. The mixing ratio, aggregate color is not always constant. That is why texture of pavement varies continuously after some distance.

Texture is an innately non-local image property. Texture is found in most natural images and therefore, the analysis of texture is an important topic of research in computer vision in the past decades [1]. Generally speaking, there are a number of filter configurations designed for texture segmentation. Most of filters are useful for particular texture but don't produce desired results when texture is varied.

Gabor filter is found helpful to analyze textures having definite orientation and frequency characteristics. Majority of prior research on Gabor filters is centered on filter-bank technique that need a filter set with predefined parameters for efficiently covering the frequency range. Such approach considerably affects classification results and is computationally expensive, so such technique is not appropriate for pavement crack detection where texture is varying all the time. In this work we develop a system for designing optimized Gabor filter for detection of pavement cracks out of pavement texture.

Additionally, optimization of Gabor filter parameters is done in semi-supervised manner while crack detection is unsupervised, because natures of cracks that can come across to this system are not known. Thus, the objective is differentiating between background textures and defective feature (crack) automatically. As the pavement cracks are randomly shaped and can be oriented in any direction, there is no specific ground truth for the comparison of effectiveness of the system. We propose simulated ground truth creation of pavement crack for optimization of Gabor filter in varying texture. Synthetic ground truth is useful in many ways like, ease in controlling parameters, fast and convenient testing of datasets.

The rest of this paper is organized as follows. Section 2 is the description and review of related work in simulated ground truth generation. In Section 3, we present the theoretical background of Gabor filter, genetic algorithm and Structural Similarity Index Measure (SSIM). We present the model of our system for optimization of Gabor filter parameters in Section 4. In Section 5, we discuss experimental results. Concluding remarks are in Section 6.

\section{LITERATURE REVIEW}

The classic standard for validation of the segmentation method is evaluation with manually done segmentation but, manual segmentation experiences the lack of reproduction and reliability, and different systems adopt different methods for manually marking part of interest. The true ground truth may require estimation from a set of manual segmentations. The condition for manual ground truth generation in such methods, is need of getting the data with all possible variations to guarantee the consistency of the training and test sets

Using simulated information has various advantages including speedy generation of dataset with low cost, management of degradations parameters and models, and convenient tests on equivalent data having same information but different background.

Simulated ground truth is formed by Nataliya et al for evaluation of performance in [2] for dirt particle counting and categorization. The aim is to create an image with a consistent 
conditions and dirt particles of various types, so that the nature and the position of every particle are known.

In [3] Serranho et al introduced a scheme to create ground truth for computation of performance metrics in perspective of OCT image processing. The study focused to set up a noisefree simulated image that imitates actual OCT data. They propose that mean square error is computed with noise-free ground truth. The computation of simulated image depends on properties of actual OCT data.

Optical Character Recognition (OCR) systems require training on the characters which are to be recognized in test data for which ground truth data is needed. In [4] Beusekom et al used synthetic ground truth for calibration of system. This data is set of character images along with their ASCII codes. Among the approaches intended for generation of ground truth of actual data, one practice is scanning documents and then utilizing them. By means of a grouping technique, the features extracted from the test document are compared with test image. For making the scheme robust to transformations, creation of simulated ground truth for training is used as a technique.

In [5], David Doermann et al discuss the generation of ground truth for OCR using the method of generating simulated document images and representative ground truth files by means of metafile information and custom print driver. In [6] Sheraz et al proposed automatic word ground truth creation of camera captured document images using a document image retrieval system. In [7] Vivek et al created and tested a method for curve detection by means of synthetically formed curves. Validation and evaluation of magnetic resonance images (MRI) segmentation is a difficult task due to shortage of reliable ground truth information. In [8] Marcel et al, suggest a method for generation of simulated multi-modal 3D brain MRI with edema and tumor. Using such ground truth images having variations in tumor dimension, position, and degree of adjacent edema, segmentation techniques can be used on images for identification. The simulated ground truth MRI is used for validation entire brain's segmentation, together with gray matter, white matter, edema and tumor. Santuari et al [9], presents a real time graphical simulator based on client-server structural design. The rendering engine, supported by a specific client application for the automatic production of objective oriented motion of synthetic characters, is used to create realistic image sequences for wide-ranging performance evaluation of computer vision algorithms for person tracking.

\section{PARAMETER OPTIMIZATION}

In this section, we discuss briefly about theoretical background of Gabor filter, and discuss method for optimization of parameters using genetic algorithm (GA) with Structural Similarity Index Measure (SSIM) as cost function.

\section{A. Gabor Filter}

Fourier analysis is the most commonly used tools in signal processing to analyze frequency characteristics of a particular signal. There are eight degrees of freedom in the 2D Gabor filter family: $x_{0}$ and $y_{0}$ specify the position of the filter in 2D spatial domain; $\mathrm{u}_{\mathrm{o}}$ and $\mathrm{v}_{\mathrm{o}}$ are modulation coordinates specify the position of the filter in $2 \mathrm{D}$ frequency domain, $\theta$ is desired orientation, $\omega$ is spatial frequency, $\psi$ is the phase offset of the modulation factor, which decides the symmetry or antisymmetry of the filter and the width (a) and the length (b) of the elliptical Gaussian (2D) envelope and the angle between orientation of sinusoidal wave vector and the two dimensional Gaussian axes [22-23]. The general form of the Gabor filter is as in (1).

$$
g(x, y)=s(x, y) w(x, y)
$$

Where the Gaussian envelops can be termed as:

$$
s(x, y)=\frac{1}{2 \pi \sigma_{x} \sigma_{y}} \exp ^{-\frac{1}{2}\left[\left(\frac{x^{\prime}}{\sigma_{x}}\right)^{2}+\left(\frac{y^{\prime}}{\sigma_{y}}\right)^{2}\right]}
$$

Here $\sigma_{x}$ and $\sigma_{y}$ are the scale factor of the neighborhood. The complex sinusoidal with phase offset $\psi$ is:

$w(x, y)=e^{j\left(2 \pi \omega_{o} x^{\prime}+\psi\right)}=\cos \left(2 \pi \omega_{o} x^{\prime}+\psi\right)+\sin \left(2 \pi \omega_{o} x^{\prime}+\psi\right)$

So the overall 2D Gabor filter

$g(x, y)=\frac{1}{2 \pi \sigma_{x} \sigma_{y}} e^{-\frac{1}{2}\left[\left(\frac{x^{\prime}}{\sigma_{x}}\right)^{2}+\left(\frac{y^{\prime}}{\sigma_{y}}\right)^{2}\right]} \times e^{j\left(2 \pi \omega_{o} x^{\prime}+\psi\right)}$

Where $x^{\prime}=x \cos \theta+y \sin \theta$ and $y^{\prime}=-x \sin \theta+y \cos \theta$. The real part of Gabor filter is as under

$g_{r}(x, y)=\frac{1}{2 \pi \sigma_{x} \sigma_{y}} e^{-\frac{1}{2}\left[\left(\frac{x^{\prime}}{\sigma_{x}}\right)^{2}+\left(\frac{y^{\prime}}{\sigma_{y}}\right)^{2}\right]} \times\left\{\cos \left(2 \pi \omega_{o}(x \cos \theta+y \sin \theta)\right)+\right.$ $\psi\}$

And the imaginary component of the complex Gabor filter is

$$
\begin{gathered}
g_{i}(x, y)=\frac{1}{2 \pi \sigma_{x} \sigma_{y}} e^{-\frac{1}{2}\left[\left(\frac{x^{\prime}}{\sigma_{x}}\right)^{2}+\left(\frac{y^{\prime}}{\sigma_{y}}\right)^{2}\right]} \times\left\{\sin \left(2 \pi \omega_{o}(x \cos \theta+y \sin \theta)\right)+\right. \\
\psi
\end{gathered}
$$

Equation (5) and (6) can be written in terms of aspect ratio $\mathrm{\gamma}=\frac{\sigma_{x}}{\sigma_{y}}$ (i.e., ellipticity of the Gabor) and wavelength $\lambda$ of the sinusoidal factor.

$$
\mathrm{g}(\mathrm{x}, \mathrm{y})=\frac{\gamma}{2 \pi \sigma^{2}} e^{-\frac{1}{2}\left[\frac{{x^{\prime}}^{2}+\gamma^{2}{y^{\prime}}^{2}}{\sigma^{2}}\right]} \times e^{j\left(\frac{2 \pi x^{\prime}}{\lambda}+\psi\right)}
$$

\section{B. Gabor Filter Power Spectrum}

The outputs of a real and an imaginary Gabor filter for each image point can be combined in a single quantity which is termed as the Gabor energy. This feature is associated with the complex cell, which is the model of orientation selective neuron in the primary visual cortex [22] and the Gabor energy is closely related to the local power spectrum and is defined in the following way;

$$
P(x, y)=E^{2}(x, y)=g_{r}{ }^{2}(x, y)+j g_{i}^{2}(x, y)
$$

A Gabor filter of wavelength of 20 , variance $=4$, phase offset $=0$ is shown in Fig. 2. And the frequency response of the Gabor filter is illustrated in Fig. 2.

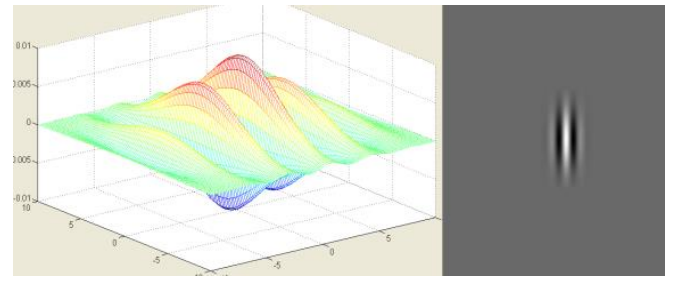

Fig. 1. Gabor filter at 0 degree orientation 


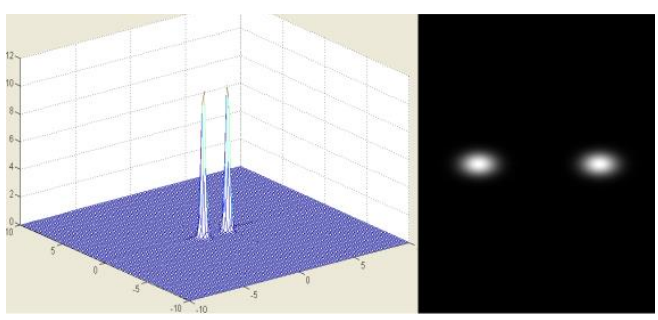

Fig. 2. Frequency response of filter in Fig. 1

\section{Genetic Algorithm}

Genetic Algorithm (GA) begins by defining the optimization variables, cost function, and associated cost. Its ending is like other optimization algorithms as well, by evaluating for convergence. However, this algorithm is relatively dissimilar. Components of GA along with their paths are shown in figure 3 . A cost function produces an output from set of input variables called a chromosome. This cost function can be a mathematical function [24]. The objective is to transform the output in a desirable manner by identifying the appropriate values for input variables. Cost function in proposed method is SSIM and input variables to be optimized for a given texture in pavement texture are variance $\sigma$ and wavelength $\lambda$. In GA literature, the term fitness is broadly used to allocate the output of the objective function. Most of the optimization literature deals with minimization of cost while fitness implies a maximization problem.

The GA begins by defining a chromosome in form of an array of variables to be optimized which are known as Genes of a chromosome.

$$
\text { Chromosome }=[\text { Gene1,Gene } 2]=[\lambda, \sigma]
$$

In this research, Gene1 is wavelength $\lambda$ and Gene 2 is variance $\sigma$, jointly are the parameters of the Gabor filter to be optimized and the desired output from GA gives the most suitable Gabor Filter to be used for crack detection in a particular texture of pavement.

Furthermore, most of the optimization problems require constraints or variable bounds while unconstrained variables can take any value. These bounds are defined in proposed algorithm section.

\section{Structural Similarity Index Measure (SSIM)}

The structural similarity Index Measure (SSIM) [25] [26] [27] is a technique for computing the similarity between two images. SSIM is formulated as a development in quality assessment. Traditional techniques such as peak signal-to-noise ratio (PSNR) and mean square error (MSE) are proven to be inconsistent with human eye perception. The advantage of SSIM over other techniques mentioned earlier (MSE and PSNR) is that SSIM consider image degradation as perceived variation in structural information while other methods estimate perceived errors. Structural information is the scheme which suggests that the pixels have strong inter-dependencies particularly when they are close in spatial domain. These dependencies contain significant information concerning the structure of the objects in an image.

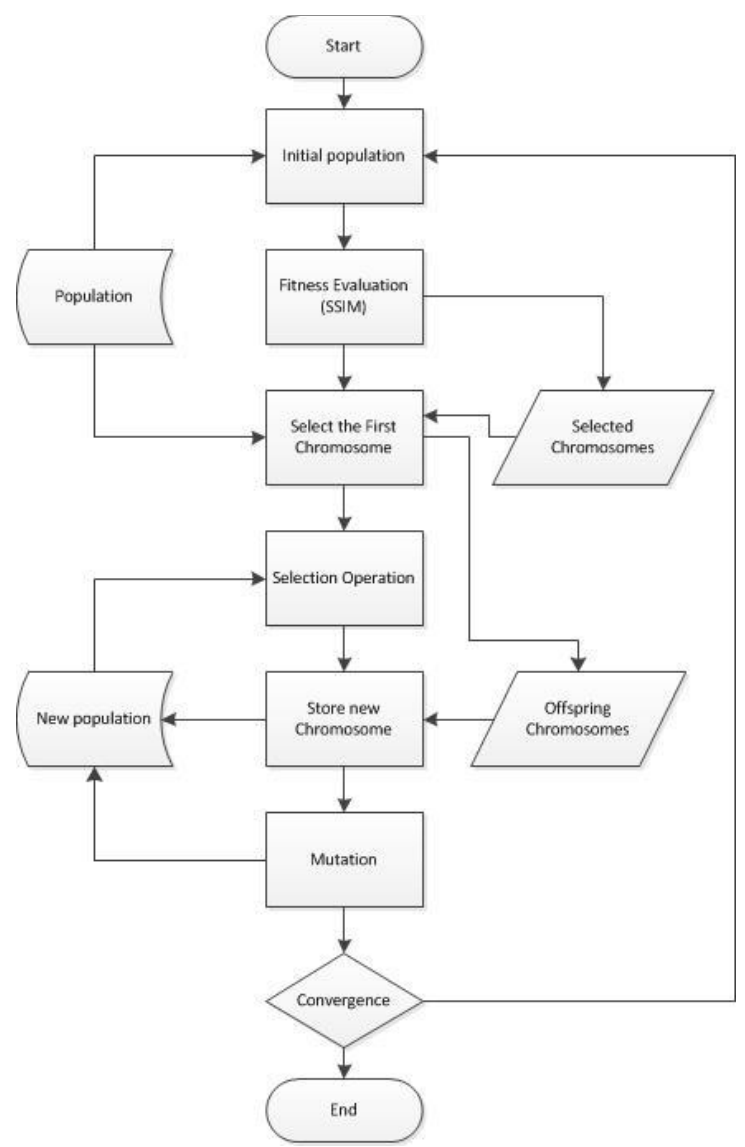

Fig. 3. Structure of Genetic Algorithm

The SSIM metric of an image is calculated on a variety of windows. To evaluate between two windows $x$ and $y$ of size $N \times N$ is:

$$
\operatorname{SSIM}(x, y)=\frac{\left(2 \mu_{x} \mu_{y}+c_{1}\right)\left(2 \sigma_{x y}+c_{2}\right)}{\left(\mu_{x}^{2}+\mu_{y}^{2}+c_{1}\right)\left(\sigma_{x}^{2}+\sigma_{y}^{2}+c_{2}\right)}
$$

Where

$\mu_{x}$ is mean and $\sigma_{x}^{2}$ is variance of $x$.

$\mu_{y}$ is mean and $\sigma_{y}^{2}$ is variance of $y$.

$\sigma_{x y}$ is the covariance between $x$ and $y$;

$C_{1}$ and $C_{2}$ are two variables for stabilizing division with weak denominator;

SSIM is applied on two images and the resultant SSIM index returns a decimal value between -1 and 1 . Value of 1 is returned if both images are exactly the same. While -1 shows complete dissimilarity.

\section{PROPOSED AlGORITHM}

In this section, the proposed method for optimization of the parameters of the Gabor filter through simulated ground truth with the aid of Genetic algorithm is discussed. The proposed system is implemented on a personal computer using MATLAB. 


\section{A. Simulated Ground Truth}

To increase the robustness of the optimization of Gabor parameters, the technique of simulated ground truth is used. This ground truth is simulated from within the texture of test image after selecting a 100 by 100 patch containing flexible pavement texture but no crack. There are relatively few low intensity pixels as compare to mean of whole patch. Low intensity pixels contain the crack present in image that is why it is avoided. This 100 by 100 patch is fused with another patch of same size which contains a synthetically created multi oriented crack as shown in figure 4.

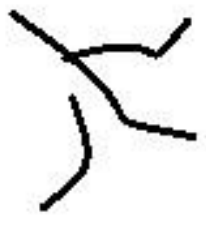

Fig. 4. Synthetic crack

The synthetic crack is arbitrary, thin as compare to original cracks and most importantly is stretched almost every direction. All these characteristics make this crack appropriate for fusion in real texture of road. As mentioned earlier that intensity values of cracks are low as compare to the rest of pavement texture but the texture is not uniform and changes continuously, the intensity must be less than the average intensity of the image. The flow diagram for simulated ground truth is shown in figure 5.

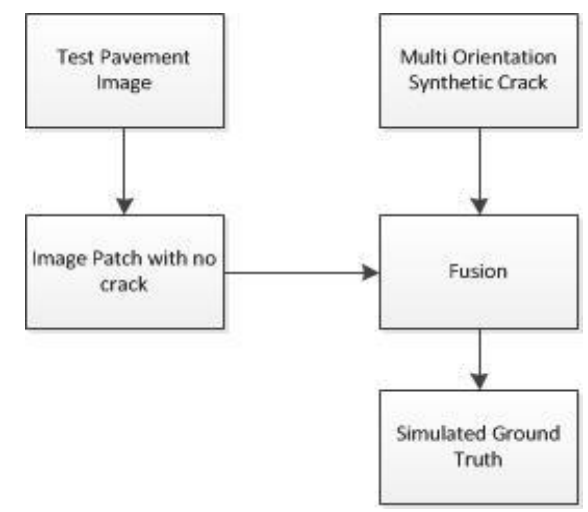

Fig. 5. The process of creating the ground truth

The input test image shown below has a multi directional crack and high coarseness. The 100 by 100 patch of pavement with no crack and the fused crack on the patch is shown in figure 6 .

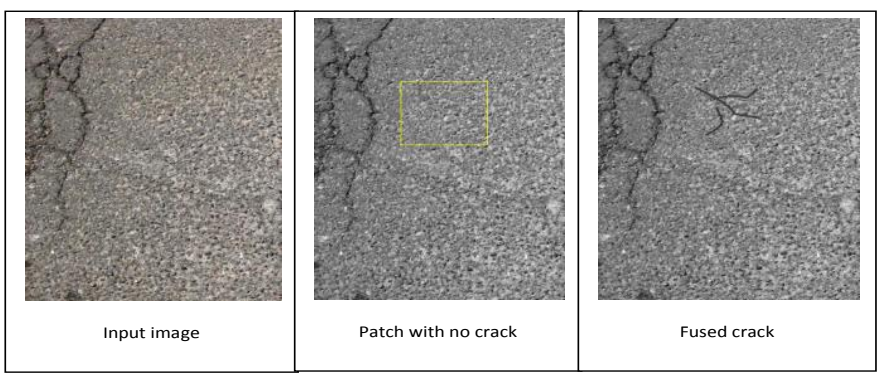

Fig. 6. Original and synthetic cracks
The 100 by 100 sized simulated ground truth is shown below in figure 7 .

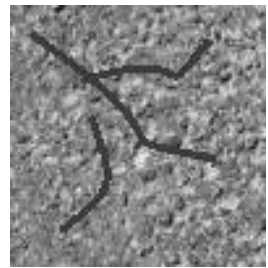

Fig. 7. Simulated Ground Truth

\section{B. Initial Population}

The designed chromosome for this research contains two genes. The first gene is the wavelength of the Gabor filter while the second gene is the variance of the Gabor filter. These two variables are the key parameters to define a Gabor filter. To get the optimized values of these variables for any type of pavement texture with lesser computation time the variable are constrained to some initial and final values. The variance of Gabor filter must be greater than 2 and lesser than 8 and the wavelength must be from 8 to 30 . These constrain are selected after intense experiments. To define these genes the binary Genetic algorithm is used. The figure 8 below shows one of the chromosomes in the initial population.

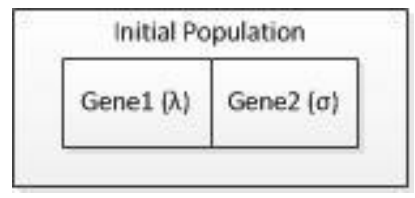

Fig. 8. Chromosomes with genes

\section{Fitness Evaluation}

As described earlier that SSIM is one of the best measure of the image structure similarities. To compute the cost of each chromosome in a generation, the Gabor filter created by each chromosome is the applied to the ground truth. The resultant image is then compared with the crack image as shown in fig [2]. Higher the similarity between the image the greater will be the SSIM values. It must be noted that the SSIM values varies from 1 to -1 .

\section{Convergence}

As soon as the stopping criteria are fulfilled, the optimized values of Gabor filter are extracted from the fittest chromosome. Now these values are fed into the crack detection algorithm to extract the crack from the original test image. As described earlier the texture of pavement varies after some distance, but it varies very little in neighboring patches. So the optimized parameters can be used for some distance and will be updated after some distance. This will increase the efficiency of system by decreasing the computation time. The figure 9 illustrates the complete scenario of the proposed method.

\section{E. Crack Detection Algorithm}

An optimized filter bank is generated using the optimized Gabor filter parameters. The filter bank contains multi orientation filter. Generally, the number of orientations depends on the application where the Gabor filter is applied. 
As the number of orientations is increased, the output is more accurate [27]. But increase in orientations also increases the computational time and complexity of the system. The Gabor filter of a given orientation is then convolved with the input preprocessed image. After the completion of convolution, the real part of the response out of the filter is threshold to produce a binary image. Finally, the binary images resulting from the differently oriented filters are combined by an OR operation to produce an output image that contains detected crack segments. The whole process is shown in flow diagram (figure 9b). In Gabor filter bank the $\lambda$ and $\sigma$ are the optimized values from GA.

\section{EXPERIMENTATION AND RESULTS}

The input image is first selected from image dataset. By using this image a simulated ground truth is created as shown previously in figure 7 . Now the ground truth and the crack image are then fed into the Genetic algorithm with the cost function based on the SSIM. Initial population size is selected randomly as 20 , selection rate 0.5 and the mutation rate is 0.01 . The optimized parameters after 25 generation are variance 2.08 and the wavelength 12.35 which are very close to the manually obtained parameters. Now the crack detection algorithm is applied to extract the crack. After experimentation, the image is thresholded at 0.7 out of 1 . The output image Fig. 10 shows the extracted crack. The Small square tiles of $10 \times 10$ pixel size are consider for crack revelation and the result are based only on that tiny region. Another extracted pavement crack is shown in figure 13.

The output of the system is computed by the precision and recall of the manually selected and optimized Gabor filter not by the values of the variance and wavelength of the optimized Gabor filter. The graph below in figure 12 shows the precision of five pavement crack images

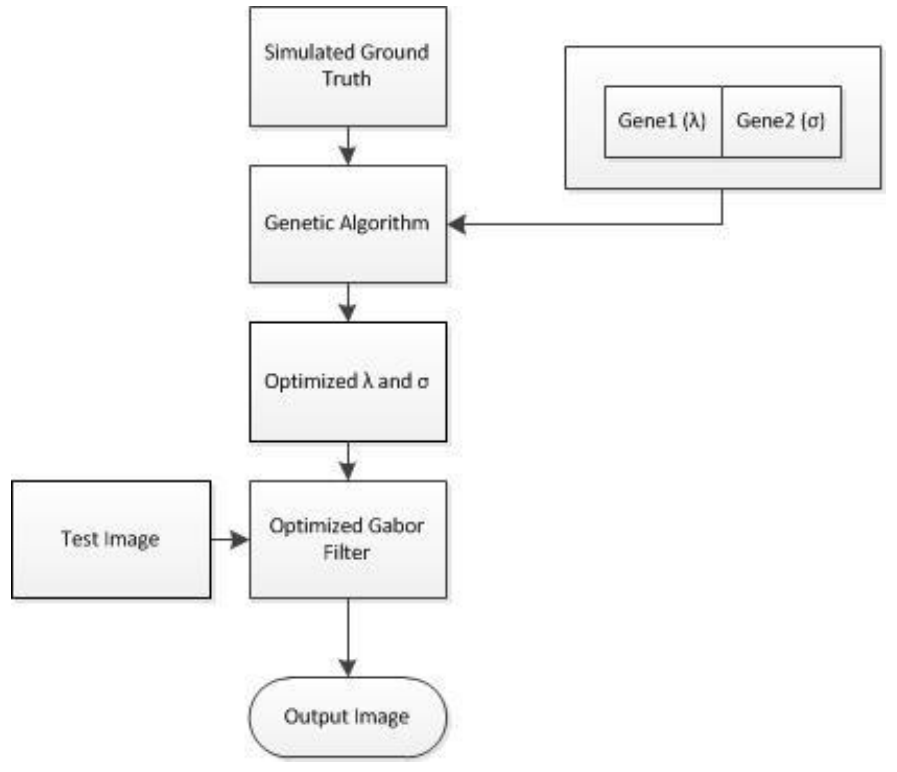

Fig. 9. a. Proposed Method Flow Chart

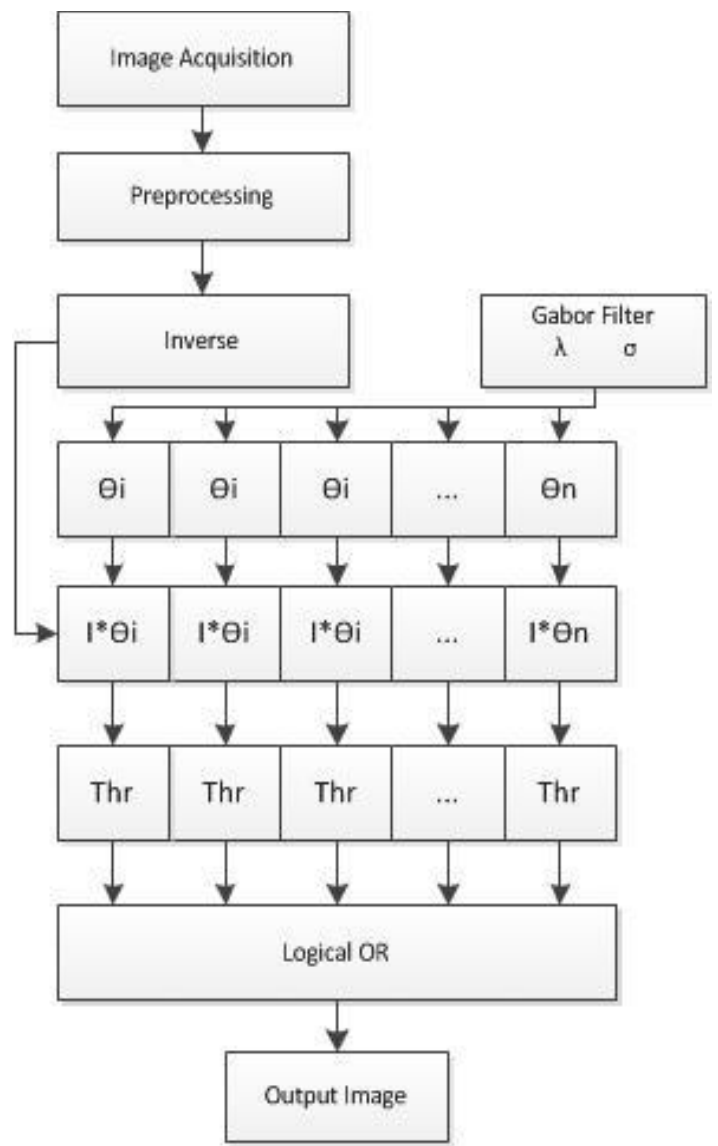

Fig. 8 . b. Crack Detection algorithm (Flow chart)

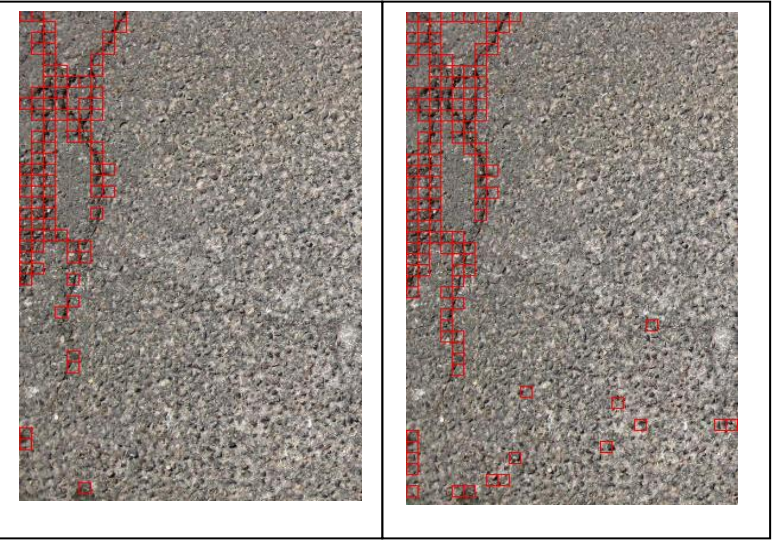

Fig. 9. Left: manually selected Gabor filter extracted crack, Right: crack extracted by optimized Gabor filter

Figure 12 shows the comparison between the output manual and optimized Gabor filter.

Table 1 shows the results for 5 images. The first two are industrial images acquired by the LRIS system. The last three are captured by the Canon IXUS 80 IScamera. The total number of crack tiles and the non-crack tiles are based on manual estimation. 


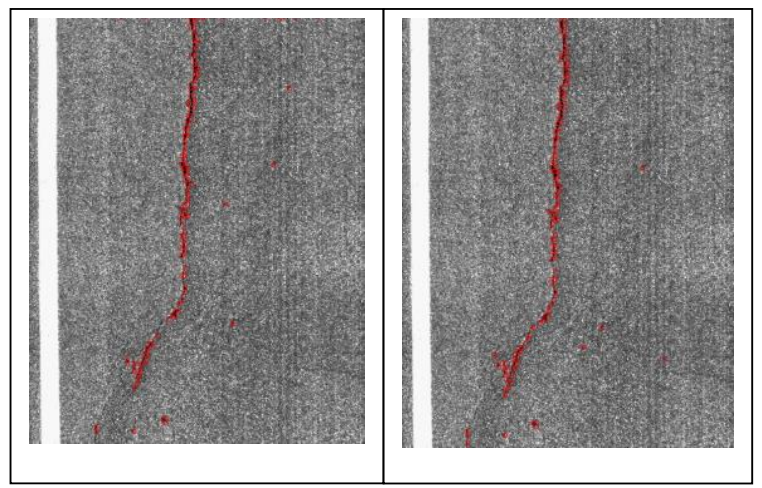

Fig. 10. Left: manually selected Gabor filter extracted crack, Right: crack extracted by optimized Gabor filter

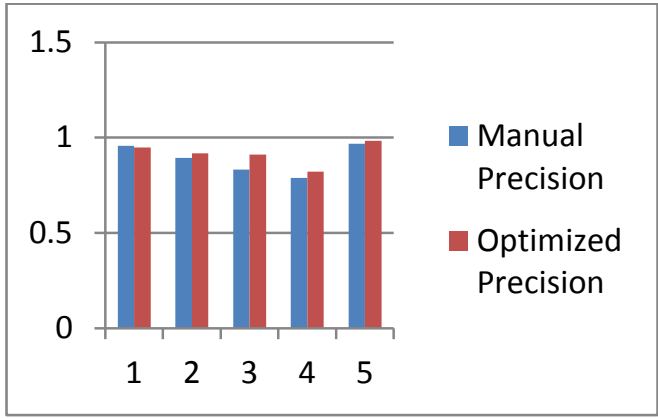

Fig. 11. Comparison between manual and Optimized Precision

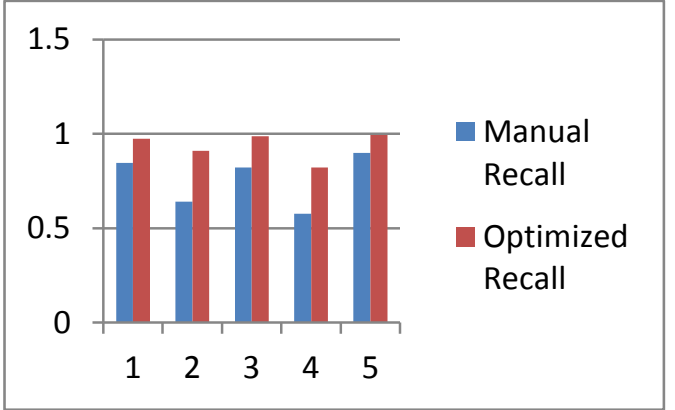

Fig. 12. Comparison between manual and Optimized Precision

\section{CONCLUSION}

This paper proposes a method to automatically optimize Gabor filter for road crack detection. The experimental results show that though the precision of the manually selected and Automated optimized Gabor filter may become similar, the execution time of optimization through our proposed method is very less and thus can be used for practical applications. As texture of pavement is changed after some interval, parameters of the Gabor filter are also updated and optimized so that pavement crack detection process can be performed smoothly and without manual calibration.

\section{REFERENCES}

[1] Lazebnik, S.; Schmid, C.; Ponce, J., "A Sparse Texture Representation Using Local Affine Regions," Pattern Analysis And Machine Intelligence, IEEE Transactions nn, Vol.27, No.8, Pp.1265,1278, Aug. 2005.

[2] N. Strokina, A. Mankki, T. Eerola, L. Lensu, J. Käyhkö, And H. Kälviäinen, "Semisynthetic Ground Truth for Dirt Particle Counting and Classification Methods", Proc. MVA, 2011, pp.215-218.
[3] Serranho, P. Maduro, C. Santos, T. Cunha-Vaz, J. Bernardes R.,"Synthetic OCT Data for Image Processing Performance Testing," 18th IEEE International Conference on Image Processing (ICIP), pp 401-404, Sept. 2011.

[4] Beusekom, Joost van; Shafait, F.; Breuel, T.M., "Automated OCR Ground Truth Generation," Document Analysis Systems, 2008. DAS '08. The Eighth IAPR International Workshop on , vol., no., pp.111,117, 16-19 Sept. 2008

[5] Gang Zi; Doermann, D., "Document image ground truth generation from electronic text," Pattern Recognition, 2004. ICPR 2004. Proceedings of the 17th International Conference on , vol.2, no., pp.663,666 Vol.2, 2326 Aug. 2004

[6] Ahmed, S.; Kise, K.; Iwamura, M.; Liwicki, M.; Dengel, A., "Automatic Ground Truth Generation of Camera Captured Documents Using Document Image Retrieval," Document Analysis and Recognition (ICDAR), 2013 12th International Conference on , vol., no., pp.528,532, 25-28 Aug. 2013

[7] Kaul, Vivek; Yezzi, Anthony; Tsai, Yichang, "Detecting Curves with Unknown Endpoints and Arbitrary Topology Using Minimal Paths," Pattern Analysis and Machine Intelligence, IEEE Transactions on , vol.34, no.10, pp.1952,1965, Oct. 2012

[8] M. Prastawa, E. Bullitt, and G. Gerig, "Synthetic Ground Truth for Validation of Brain Tumor MRI Segmentation", Proc. MICCAI, 2005, pp.26-33.

[9] Santuari and O. Lanz and R. Brunelli, "Synthetic movies for computer vision applications:, Proc. 3rd IASTED International Conference: Visualization, Imaging, and Image Processing (VIIP 2003) pp. 1-6

[10] R. W. Lucky, "Automatic equalization for digital communication," Bell Syst. Tech. J., vol. 44, no. 4, pp. 547-588, Apr. 1965.

[11] De Valois, R. L., Albrecht, D. G. and Thorell, L. G.,"Spatial-frequency selectivity of cells in macaque visual cortex", Vision Research 22, 545559 (1982)

[12] S. P. Bingulac, "On the compatibility of adaptive controllers ," in Proc. 4th Annu. Allerton Conf. Circuits and Systems Theory, New York, 1994, pp. 8-16.

[13] G. R. Faulhaber, "Design of service systems with priority reservation," in Conf. Rec. 1995 IEEE Int. Conf. Communications, pp. 3-8.

[14] Doyle, W.D., "Magnetization reversal in films with biaxial anisotropy," Magnetics, IEEE Transactions on , vol.2, no.2, pp.68,73, Jun 1966

[15] Juette, G.W.; Zaffanella, L.E., "Radio Noise Currents and Audible Noise on Short Sections of UHV Bundle Conductors," Power Apparatus and Systems, IEEE Transactions on, vol.PAS-89, no.5, pp.902,913, May 1970

[16] J. G. Kreifeldt, "An analysis of surface-detected EMG as an amplitudemodulated noise," presented at the 1989 Int. Conf. Medicine and Biological Engineering, Chicago, IL.

[17] J. Williams, "Narrow-band analyzer", Ph.D. dissertation, Dept. Elect. Eng., Harvard Univ., Cambridge, MA, 1993.

[18] N. Kawasaki, "Parametric study of thermal and chemical nonequilibrium nozzle flow," M.S. thesis, Dept. Electron. Eng., Osaka Univ., Osaka, Japan, 1993.

[19] J. P. Wilkinson, "Nonlinear resonant circuit devices (Patent style)," U.S. Patent 3624 12, July 16, 1990.

[20] Jain, A. K., Ratha,N. K.; Lakshmanan, S., “Object detection using Gabor filters", Pattern Recognition 30, 295-309 (1997).

[21] Porat, M. andZeevi, Y. Y.,"The generalized Gabor scheme of image representation in biological and machine vision", IEEE Trans. Pattern Analysis Mach. Intell. 10, 452-468 (1988)

[22] Daugman, J. (1980)., "Two-dimensional analysis of cortical receptive field profiles", Vision Research, 20, 846-856

[23] Daugman, J. (1985), "Uncertainty relation for resolution in space, spatial frequency, and orientation optimized by two-dimensional visual cortical filters", Journal of the Optical Society of America-A, 2(7), 1160-1169.

[24] Shi, Xian-Jun; Hong Lei, "A Genetic Algorithm-Based Approach for Classification Rule Discovery," Information Management, Innovation Management and Industrial Engineering, 2008. ICIII '08. International Conference on , vol.1, no., pp.175,178, 19-21 Dec. 2008 
[25] Zhou Wang; Bovik, A.C.; Sheikh, H.R.; Simoncelli, E.P., "Image quality assessment: from error visibility to structural similarity," Image Processing, IEEE Transactions on, vol.13, no.4, pp.600,612, April 2004.

[26] Salman, M. Mathavan, S.; Kamal, K.; Rahman, M., "Pavement crack detection using the Gabor filter," Intelligent Transportation Systems -
(ITSC), 2013 16th International IEEE Conference on , vol., no., pp.2039,2044, 6-9 Oct. 2013

[27] Casti, P., Mencattini, A., Salmeri, M., \& Rangayyan, R. M., “Analysis of Structural Similarity in Mammograms for Detection of Bilateral Asymmetry," Medical Imaging, IEEE Transactions on, 34(2), 662-671, 2015.

TABLE I. RESULT FOR 5 IMAGES

\begin{tabular}{|c|c|c|c|c|c|c|c|c|c|}
\hline \multirow{2}{*}{$\begin{array}{l}\text { Img } \\
\text { No. }\end{array}$} & \multirow{2}{*}{$\begin{array}{l}\text { Crack } \\
\text { Tiles }\end{array}$} & \multicolumn{2}{|c|}{ True Positives } & \multicolumn{2}{|c|}{ False Positives } & \multicolumn{2}{|c|}{ False Negatives } & \multicolumn{2}{|c|}{ True Negatives } \\
\hline & & Manual & Optimized & Manual & Optimized & Manual & Optimized & Manual & Optimized \\
\hline 1 & 155 & 131 & 148 & 6 & 8 & 24 & 4 & 11181 & 11179 \\
\hline 2 & 145 & 93 & 132 & 11 & 12 & 52 & 13 & 1120 & 1119 \\
\hline 3 & 73 & 60 & 72 & 12 & 7 & 13 & 1 & 1367 & 1372 \\
\hline 4 & 45 & 26 & 37 & 7 & 8 & 19 & 8 & 432 & 431 \\
\hline 5 & 173 & 151 & 169 & 5 & 3 & 17 & 1 & 11169 & 11169 \\
\hline
\end{tabular}

